\title{
BMJ Open Endoscopic ultrasound versus endoscopic retrograde cholangiopancreatography-guided biliary drainage for primary decompression of malignant biliary obstruction: protocol for a systematic review and meta-analysis of randomised controlled trials
}

Zheng Jin, ${ }^{\odot}$ Yaping Wei, Huapeng Lin, Haitao Huang, Wen Lv, Xiaofeng Zhang

To cite: Jin Z, Wei Y, Lin $\mathrm{H}$, et al. Endoscopic ultrasound versus endoscopic retrograde cholangiopancreatographyguided biliary drainage for primary decompression of malignant biliary obstruction: protocol for a systematic review and meta-analysis of randomised controlled trials. BMJ Open 2019;9:e028156. doi:10.1136/ bmjopen-2018-028156

- Prepublication history and additional material for this paper are available online. To view please visit the journal (http:// dx.doi.org/10.1136/bmjopen2018-028156).

ZJ, YW and $\mathrm{HL}$ contributed equally.

Received 24 November 2018 Revised 3 April 2019

Accepted 23 May 2019

Check for updates

(c) Author(s) (or their employer(s)) 2019. Re-use permitted under CC BY-NC. No commercial re-use. See rights and permissions. Published by BMJ.

For numbered affiliations see end of article.

Correspondence to Dr Xiaofeng Zhang; zxf837@tom.com

\section{ABSTRACT}

Introduction Current evidence supporting the utility of endoscopic ultrasound-guided biliary drainage (EUS-BD) as a first-line treatment option for malignant biliary obstruction (MBO) is limited. We plan to provide a systematic review and meta-analysis to compare the performance of EUS-BD and endoscopic retrograde cholangiopancreatography-guided biliary drainage (ERCPBD) as primary palliation of MBO.

Methods and analysis Randomised controlled trials evaluating EUS-BD versus ERCP-BD in primary drainage of MBO will be searched in MEDLINE, EMBASE, Web of Science, the Cochrane Library, ClinicalTrials.gov and Google Scholar, from database inception to 31 October 2018. Data on study design, participant characteristics, intervention details and outcomes will be extracted. Primary outcomes to be assessed are technical and clinical success. Secondary outcomes include adverse events, stent patency, stent dysfunction, reinterventions, procedure duration and overall survival. Study quality will be assessed using the Cochrane Risk of Bias Tool. Meta-analysis will be performed using RevMan V.5.3 statistical software. Data will be combined with a random effect model. The results will be presented as a risk ratio for dichotomous data, weighted mean difference for continuous data and HR for time-to-event data. Publication bias will be visualised using funnel plots.

Ethics and dissemination This study will not use primary data, and therefore, formal ethical approval is not required. The findings will be disseminated through peer-reviewed journals and committee conferences.

PROSPERO registration number CRD42018117040

\section{INTRODUCTION}

Not uncommonly, malignant biliary obstruction (MBO) is diagnosed at an advanced
Strengths and limitations of this study

This is the first meta-analysis comparing endoscopic ultrasound-guided biliary drainage- with endoscopic retrograde cholangiopancreatography-guided biliary drainage for primary drainage of malignant biliary obstruction.

- The study selection, data extraction and quality assessment will be performed independently by two researchers.

- We will calculate time-to-event outcomes using HRs, in contrast to other meta-analyses using risk ratios or weighted mean differences.

- We will be able to comprehensively survey the literature and identify areas where further study may be required.

A possible weakness may be the quality of the trials we include.

stage when treatment is mainly palliative. Endoscopic retrograde cholangiopancreatography-guided biliary drainage (ERCP-BD) has been the most commonly used technique for the palliation of MBO. ${ }^{1}$ However, a wide range of postprocedure complications has continued to pose a serious challenge. ${ }^{2}$ In addition, patients with MBO may be accompanied by duodenal invasion and altered anatomy from the previous surgeries, which could increase ERCP difficulty. ${ }^{3}$

Since first reported by Giovannini et al, ${ }^{4}$ endoscopic ultrasound-guided biliary drainage (EUS-BD) has emerged as an alternative procedure to percutaneous transhepatic biliary drainage (PTBD) after failed 
ERCP. $^{5-8}$ A recent meta-analysis evaluating EUS-BD reported cumulative technical success and adverse events of $94.71 \%$ and $23.32 \%$, respectively. ${ }^{9}$ With increasing availability and familiarity with this procedure, several studies have compared EUS-BD versus ERCP-BD for primary biliary decompression for $\mathrm{MBO} .{ }^{10-12}$ These studies have reported variable results and were limited because of small sample sizes. We had planned to conduct a meta-analysis to compare the performance of EUS-BD with ERCP-BD as primary treatment in relieving MBO.

\section{METHODS}

The review will be performed according to the recommendations specified in the Cochrane Handbook for Intervention Reviews. ${ }^{13}$ The reporting of the review will follow the Preferred Reporting Items for Systematic Reviews and Meta-Analyses (PRISMA) statement. ${ }^{14}$

\section{Criteria for considering studies for this review}

Eligibility criteria are established in terms of the population, intervention, comparison, outcome, study design framework. Studies will be selected according to the following criteria:

\section{Participants}

Included studies will involve patients presenting with $\mathrm{MBO}$ and initially undergoing endoscopic drainage, with no age limitation. Both distal and hilum MBO will be included. There will be no restrictions on aetiology, which include, but are not limited to, pancreatic cancer, cholangiocarcinoma, gallbladder cancer, ampulla of vater cancer and metastasis. Patients with benign biliary diseases, and those with EUS-BD performing as a salvage procedure for failed ERCP will be excluded.

\section{Interventions/comparison}

The intervention comparisons are EUS-BD versus ERCPBD. EUS-BD can be performed in several ways, choledochoduodenostomy, hepaticogastrostomy, antegrade procedure and rendezvous technique. ${ }^{15}$ All these methods of EUS-BD will be included except for rendezvous technique. Because the rendezvous approach is a cross-over technique using EUS to pass a guidewire via the papilla to perform an ERCP. There will be no restrictions on stent type (metal/plastic stent), dilation device (dilation catheter/cystotome/balloon), and whether patient has an indwelling duodenal stent or not.

\section{Outcomes}

There are two primary outcomes for this study: technical success (defined as successful stent placement as determined endoscopically or radiographically) and clinical success (defined as reduction of total serum bilirubin levels to less than half of the preoperative level within 4 weeks). ${ }^{10}$

There are six secondary outcomes: (1) adverse events: total, pancreatitis, cholangitis, cholecystitis, bleeding and bile peritonitis; (2) stent patency (HR) for interval from initial insertion to recurrence of obstruction); (3) stent dysfunction: stent occlusion, stent migration and tumour in/overgrowth; (4) reinterventions; (5) procedure duration and (6) overall survival (HR for death).

\section{Study design}

Only randomised controlled trials (RCTs) will be included. Unpublished trials and abstracts will be included if the methodology and data are accessible. We will only include studies that are presented in English language due to constraints in translational resources.

Exclusion criteria will be: (1) studies without a comparative arm of ERCP-BD; (2) observational studies, case reports, reviews, editorials and letters to editor; (3) duplicate studies, in vitro studies or animal studies and (4) no data on any of the primary or secondary outcomes.

\section{Search methods for identification of studies}

\section{Electronic searches}

Two investigators (ZJ and YW) will independently search MEDLINE, EMBASE, Web of Science, the Cochrane Library, ClinicalTrials.gov and Google Scholar, for all entries through 31 October 2018. The search strategies will be decided on after a discussion among all reviewers. The primary search strategy will be used for PubMed MEDLINE (online supplementary appendix I). Modifications to the search strategy will be made for other databases. We will assess eligibility of the retrieved articles by title and abstract using predetermined inclusion criteria. If this information is insufficient for eligibility assessment, we will review the full article. If any up-to-date evidence is published during the review period, we will evaluate the eligibility of each study and consider its addition to the analysis.

\section{Searching other resources}

To further increase the robustness of the literature search, a manual recursive search of the reference sections of the retrieved articles, as well as the related articles option in PubMed, will be carried out to identify other potentially relevant articles.

\section{Data collection and analysis \\ Selection of studies}

Decisions about study inclusion and exclusion will be made independently by two investigators (ZJ and $\mathrm{YW}$ ). Disagreements will be resolved by consensus after a mutual discussion. The details of the study selection procedure are shown in a PRISMA flow chart. (figure 1)

\section{Data extraction and management}

Two investigators (YW and HL) will independently extract the appropriate data onto a data collection form (online supplementary appendix II). The following variables will be contained in the collection form: country and year of the study, study design, patient demographics and clinical characteristics, methods of EUS-BD, types of stents, technical success, clinical success, procedure duration, stent patency, stent dysfunction, reinterventions, adverse 


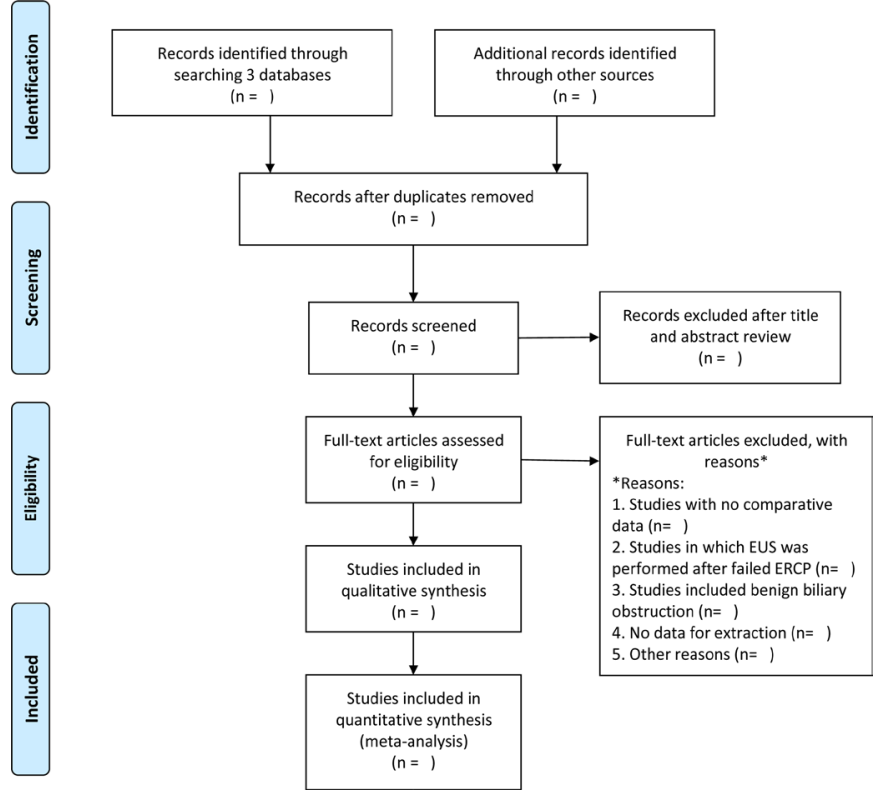

Figure 1 Flow diagram of the study selection process. ERCP, endoscopic retrograde cholangiopancreatography; EUS, endoscopic ultrasound.

events, overall survival and follow-up information. When necessary data are not included in the published studies, the corresponding authors will be contacted for additional information. If there is no reply, we will analyse only the available data. If there are no data on any of the primary or secondary outcomes, those studies will be excluded from the meta-analyses.

\section{Assessment of risk of bias in included studies}

We will assign two independent investigators (YW and HL) to appraise methodological quality of the included trials with the Cochrane Collaboration's tool for assessing risk of bias. ${ }^{16}$ The tool appraises existence of selection bias by assessing methods of randomisation and allocation concealment, performance and detection of biases by checking blinding of personnel and outcome assessment, and attrition and reporting bias by evaluating incomplete and selective data reporting. Each of the items is assigned a judgement of high, low or unclear risk.

\section{Data synthesis}

The HRs for time-to-event outcomes (stent patency and overall survival) will be calculated using the Excel sheet published by Tierney et al, ${ }^{17}$ based on Parmar et al $\mathrm{s}$ method of data extraction ${ }^{18}$ from Kaplan-Meier curves. Weighted mean differences (WMDs) will be calculated for continuous variables. Medians will be used if means are not available and SDs will be calculated or imputed when possible. ${ }^{19}$ Risk ratios (RRs) will be calculated for categorical variables. Owning to the assumption of inherently various study scenarios and study populations, a random effects model for all analyses will be assumed. ${ }^{13}$ Heterogeneity among studies will be assessed by calculating the $\mathrm{I}^{2}$ statistics whereby $\mathrm{I}^{2}<25 \%$ indicates no heterogeneity, $25 \% \leq \mathrm{I}^{2}<50 \%$ indicates mild heterogeneity, $50 \% \leq$
$\mathrm{I}^{2}<75 \%$ indicates moderate heterogeneity and $\mathrm{I}^{2} \geq 75 \%$ indicates strong heterogeneity. ${ }^{20}$ We had planned that if sufficient studies $(\geq 10)$ are included in the analysis of primary outcomes, we would construct funnel plots to evaluate publication bias, ${ }^{13}$ otherwise, Egger's test will be applied. ${ }^{21}$ All statistical analyses will be performed using Review Manager V.5.3 (Cochrane Collaboration, 2014).

\section{Subgroup analyses}

In the case of possible strong heterogeneity, we will explore the possible sources using subgroup and meta-regression analyses. Subgroup analyses will be carried out based on geographical location, publication form, study design, location of biliary obstruction, indwelling duodenal stent, EUS-BD technique, stent type and definitions of adverse event. For those subgroups with only one study included, subgroup analyses will not be performed.

\section{Sensitivity analysis}

We will carry out a sensitivity analysis by systematically removing every study and checking the pooled results for the remaining studies to see if there is any significant change in test performance.

\section{Patient and public involvement}

Because the collected data within this systematic review and meta-analysis originates from previously published studies, patients and the general public were not involved in the development of the research question or choice of outcome measures that we wanted to assess.

\section{DISCUSSION}

ERCP-BD has been a generally preferred treatment for inoperable $\mathrm{MBO} .{ }^{22-24}$ Conventionally, when ERCP fails for achieving biliary drainage, patients undergo PTBD and EUS-BD. ${ }^{5825}$ For primary drainage of MBO, several studies have investigated EUS-BD versus ERCP-BD showing different results. $^{10-12} \mathrm{~A}$ recent meta-analysis $^{9}$ reported that EUS-BD may not be used as an initial modality for relieving biliary obstruction, however, none of the included studies were direct comparative. We, therefore, propose a meta-analysis to pool the evidence to evaluate the performance of EUS-BD versus ERCP-BD.

One strength of our meta-analysis will be that stent patency and overall survival will be calculated using HRs, in contrast to other meta-analyses using RRs or WMDs. ${ }^{26}$ Because the included studies had various length of follow-up, and the events might not occur in some patients at the end of study. For these time-to-event outcomes, the most appropriate way of analysis is to use methods of survival analysis and express the intervention effect as an HR. ${ }^{13}$ This will be the first meta-analysis of RCTs comparing EUS-BD with ERCP-BD for primary drainage of MBO. The results of this study will influence decision-making for unresectable $\mathrm{MBO}$, assist in future guideline development and guide future research endeavours. 


\section{DISSEMINATION}

We will disseminate the findings of our work through conference presentations and a peer-reviewed publication.

Author affiliations

Affiliated Hangzhou First People's Hospital, Zhejiang University School of Medicine, Hangzhou, China

Contributors $\mathrm{XZ}$ is the guarantor. ZJ drafted the manuscript protocol. YW, HL and WL contributed to the development of the selection criteria, article screening strategy, risk of bias assessment strategy and data extraction criteria. ZJ developed the search strategy. HH provided statistical expertise. All authors read, provided feedback and approved the final protocol.

Funding The authors have not declared a specific grant for this research from any funding agency in the public, commercial or not-for-profit sectors.

Competing interests None declared.

Patient consent for publication Not required.

Ethics approval Ethics approval will not be required because all analyses in the present study will be performed based on data from published studies.

Provenance and peer review Not commissioned; externally peer reviewed.

Open access This is an open access article distributed in accordance with the Creative Commons Attribution Non Commercial (CC BY-NC 4.0) license, which permits others to distribute, remix, adapt, build upon this work non-commercially, and license their derivative works on different terms, provided the original work is properly cited, appropriate credit is given, any changes made indicated, and the use is non-commercial. See: http://creativecommons.org/licenses/by-nc/4.0/.

\section{REFERENCES}

1. Moss AC, Morris E, Leyden J, et al. Do the benefits of metal stents justify the costs? A systematic review and meta-analysis of trials comparing endoscopic stents for malignant biliary obstruction. Eur $J$ Gastroenterol Hepatol 2007:19:1119-24.

2. Almadi MA, Barkun A, Martel M. Plastic vs. self-expandable metal stents for palliation in malignant biliary obstruction: a series of metaanalyses. Am J Gastroenterol 2017;112:260-73.

3. Dhir V, Itoi T, Khashab MA, et al. Multicenter comparative evaluation of endoscopic placement of expandable metal stents for malignant distal common bile duct obstruction by ERCP or EUS-guided approach. Gastrointest Endosc 2015;81:913-23.

4. Giovannini M, Moutardier V, Pesenti C, et al. Endoscopic ultrasoundguided bilioduodenal anastomosis: a new technique for biliary drainage. Endoscopy 2001;33:898-900.

5. Poincloux L, Rouquette O, Buc E, et al. Endoscopic ultrasoundguided biliary drainage after failed ERCP: cumulative experience of 101 procedures at a single center. Endoscopy 2015;47:794-801.

6. Kawakubo $\mathrm{K}$, Isayama $\mathrm{H}$, Kato $\mathrm{H}$, et al. Multicenter retrospective study of endoscopic ultrasound-guided biliary drainage for malignant biliary obstruction in Japan. $J$ Hepatobiliary Pancreat Sci 2014;21:328-34.

7. Lee TH, Choi JH, Park doH, et al. Similar efficacies of endoscopic ultrasound-guided transmural and percutaneous drainage for malignant distal biliary obstruction. Clin Gastroenterol Hepatol 2016;14:1011-9.

8. Sharaiha RZ, Khan MA, Kamal F, et al. Efficacy and safety of EUSguided biliary drainage in comparison with percutaneous biliary drainage when ERCP fails: a systematic review and meta-analysis. Gastrointest Endosc 2017;85:904-14.

9. Wang K, Zhu J, Xing L, et al. Assessment of efficacy and safety of EUS-guided biliary drainage: a systematic review. Gastrointest Endosc 2016;83:1218-27.

10. Park JK, Woo YS, Noh DH, et al. Efficacy of EUS-guided and ERCP-guided biliary drainage for malignant biliary obstruction: prospective randomized controlled study. Gastrointest Endosc 2018;88:277-82.

11. Paik WH, Lee TH, Park DH, et al. EUS-Guided Biliary Drainage Versus ERCP for the primary palliation of malignant biliary obstruction: a multicenter randomized clinical trial. $A m \mathrm{~J}$ Gastroenterol 2018;113:987-97.

12. Bang JY, Navaneethan U, Hasan M, et al. Stent placement by EUS or ERCP for primary biliary decompression in pancreatic cancer: a randomized trial (with videos). Gastrointest Endosc 2018;88:9-17.

13. Higgins JP, Green S. Cochrane handbook for systematic reviews of interventions, 2011.

14. Moher D, Liberati A, Tetzlaff J, et al. Preferred reporting items for systematic reviews and meta-analyses: the PRISMA statement. BMJ 2009;339:b2535.

15. Baars JE, Kaffes AJ, Saxena P. EUS-guided biliary drainage: a comprehensive review of the literature. Endosc Ultrasound 2018;7:4-9.

16. Higgins JP, Altman DG, Gøtzsche PC, et al. The Cochrane Collaboration's tool for assessing risk of bias in randomised trials. BMJ 2011;343:d5928.

17. Tierney JF, Stewart LA, Ghersi D, et al. Practical methods for incorporating summary time-to-event data into meta-analysis. Trials 2007;8:16.

18. Parmar MK, Torri V, Stewart L. Extracting summary statistics to perform meta-analyses of the published literature for survival endpoints. Stat Med 1998;17:2815-34.

19. Hozo SP, Djulbegovic B, Hozo I. Estimating the mean and variance from the median, range, and the size of a sample. BMC Med Res Methodol 2005;5:13.

20. Higgins JP, Thompson SG. Quantifying heterogeneity in a metaanalysis. Stat Med 2002;21:1539-58.

21. Egger M, Davey Smith G, Schneider M, et al. Bias in meta-analysis detected by a simple, graphical test. BMJ 1997;315:629-34.

22. Speer AG, Cotton PB, Russell RC, et al. Randomised trial of endoscopic versus percutaneous stent insertion in malignant obstructive jaundice. Lancet 1987;2:57-62.

23. Inamdar S, Slattery E, Bhalla R, et al. Comparison of adverse events for endoscopic vs percutaneous biliary drainage in the treatment of malignant biliary tract obstruction in an inpatient national cohort. JAMA Oncol 2016;2:112-7.

24. Pu LZ, Singh R, Loong CK, et al. Malignant biliary obstruction: evidence for best practice. Gastroenterol Res Pract 2016;2016:3296801.

25. Moole H, Dharmapuri S, Duvvuri A, et al. Endoscopic versus percutaneous biliary drainage in palliation of advanced malignant hilar obstruction: a meta-analysis and systematic review. Can $J$ Gastroenterol Hepatol 2016;2016:1-8.

26. Uemura RS, Khan MA, Otoch JP, et al. EUS-guided choledochoduodenostomy versus hepaticogastrostomy. J Clin Gastroenterol 2017;52:1. 